\title{
Security CT After-sales Service System Design Based on SSI Framework Yongchang Ren ${ }^{\mathrm{a}}$, Yongzhe $\mathrm{Ma}^{\mathrm{b}}$, Jiahui Wang ${ }^{\mathrm{c}}$ \\ School of Information Science and Technology, Bohai University, Jinzhou, 121013, China \\ a1213552916@qq.com, b1125444268@qq.com, ㄷ2431108@qq.com
}

Keywords: SSI framework; system architecture design; preliminary design; data structure design

\begin{abstract}
After-sales service is an important index for users to evaluate products, it is necessary to strengthen the quality and efficiency of after sales service to improve customer satisfaction. In view of the difficult problems in the development of security CT after-sales service system, this article designs the system based on SSI framework. First of all, the paper expounds SSI framework structure, the composition is described in detail; secondly, on the basis of SSI framework, adds a layer of interface layer, designs the system architecture; then, designs data storage structure which is based on MySQL database management system; finally, designs the system function, including seven functional modules of equipment repair, equipment maintenance, equipment security, spare parts application, spare parts inventory information query, spare parts in-out storage management and basic data management. Adopting the design method of this paper can effectively improve the efficiency and quality of system development.
\end{abstract}

\section{Introduction}

After-sales service is a kind of service activities which is provided for customers after the commodity is sold, a set of perfect after-sales service system can improve the customer satisfaction of products, enhance the brand influence, which is the important guarantee of product quality. the proportion of the profits of after-sales service occupies is more and more high, which is converting from daily maintenance work to providing customers with a variety of value-added services [1]. With the traditional manual management mode, because more staff and leadership are involved, business processing and approval process are relatively complex, human and other factors cause some problems such as the error of information transmission, work delay, long maintenance work response time, high customer complaint rate and so on. According to the characteristics of security CT after-sales service business, the paper builds a security CT after-sales service system, and carries on the classification management of the business, the staff can login the system to deal with daily business at anytime and anywhere, which can effectively solve the above problems, achieve a paperless after-sales service [2]. Truly provide users with efficient and convenient after-sales service experience, help enterprises to improve product popularity, win the competitive advantage.

After sufficient demand researches, this article designs the system on the basis of SSI integration framework, mainly setting the system function and performance which is based on the demand model, confirming the design model of software system [3]. Including the system architecture design, preliminary design and data storage structure design, which provides a reference for the later system development.

\section{SSI Framework}

SSI framework is composed of three kinds of frameworks of struts, spring and ibatis, applied respectively to the presentation layer, business logic layer and data persistence layer, which is a typical of three layer development architecture. The limitation is it cannot make full use of the advantages of each framework to improve the speed of development, reduce costs, and achieve the goal of high cohesion and low coupling of system. MVC has the advantages of low coupling, high reusability, applicability, low cost, rapid deployment, maintainability and so on, which is conducive to software engineering management [4]. Therefore, this article adopts MVC software design mode, and combines with the respective features of three kinds of framework to build SSI integration 
framework. Specific fusion method is as follows: with the struts framework as the basic infrastructure, integrated with the spring framework, applied to the presentation layer; according to the URL users invoke can quickly mapping corresponding action through the struts-config.xml configuration file, the data is returned after performing the corresponding operation, using js, CSS and Ajax technology to write dynamic pages to interact with users [5]. Integrating spring and ibatis framework, applied to the business logic layer, mapping service class interface in the sqlService.xml configuration file, transferring the relevant interface implementation method to complete data encapsulation and business logic processing. Data persistence layer is an important part of the model, deploying ibatis framework mainly is to keep communication with the database management system, using the mapping mechanism to realize the basic operation of the data in the dao.xml configuration file, complete the data access service [6], achieve the data persistence. Because of the limited space, this paper does not make a detailed description of the specific implementation process, can refer to system architecture design for reference.

\section{System Architecture Design}

The system structure of this paper adopts MVC software design pattern and joins a layer of interface layer on the basis of SSI three-layer framework, which is made up of service and serviceImpl, respectively stored in sqlservice.xml and sqlmap.xml file, which implements the separation of business logic processing and data persistence, avoids data transfer confusion and loss. The system structure can furthest improve code reuse, speed up development speed. Four layer system structure from top to bottom, respectively for the presentation layer, business logic layer, interface layer and data persistence layer, among them, the presentation layer mainly uses technology such as JavaScript, css, Ajax and others to display the interface the user interacts with the system, by specifying the URL obtains the corresponding method to achieve functional response; the business logic layer consists of Action and ActionForm objects. Action completes the business logic processing users request data, ActionForm components complete data encapsulation and implement the interact with background by the interface layer [7], method invocation mechanism can be configured in the struts.xml file; the data persistence layer receives data through the interface layer, according to the data mapping mechanism completes data CRUD operations, and encapsulates data, and parsed through the browser, presented to the user. In the process that the user interacts with the system, the data circularly flows according to the above process, which guarantees data real-time. System architecture is shown in Fig. 1.

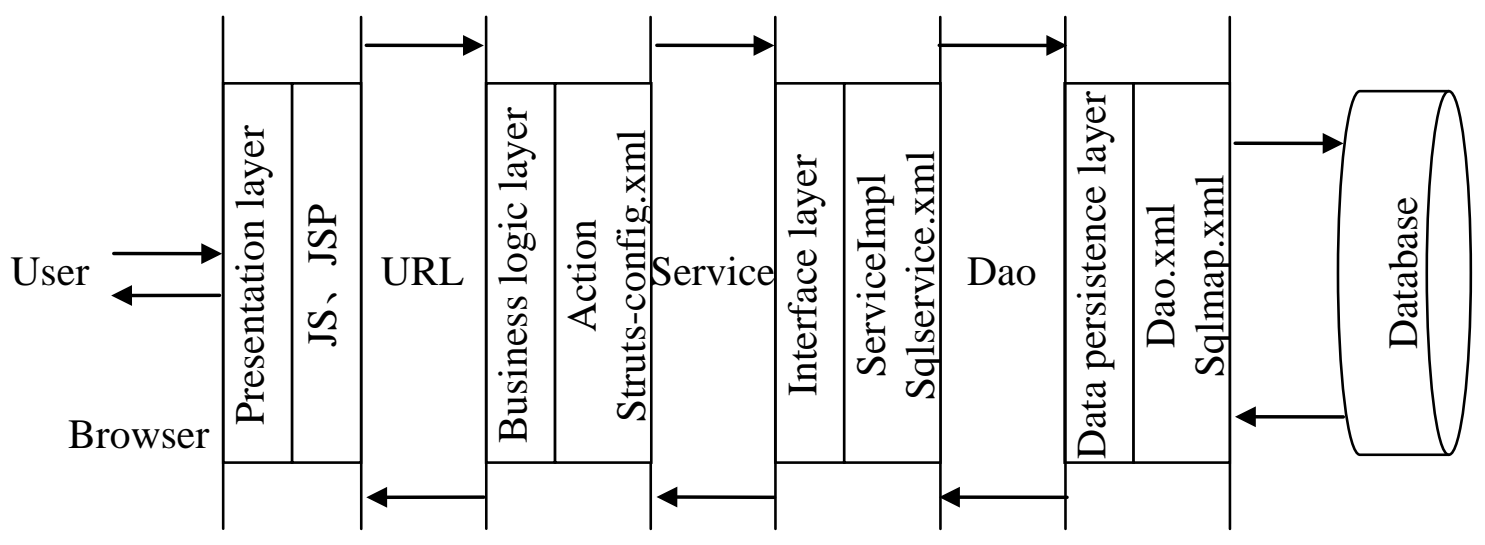

Fig. 1. Security CT after-sales service system structure 


\section{Preliminary Design}

In the software development cycle, after the requirement analysis is completed, the next step work is to carry on the system preliminary design, which is based on the requirement analysis, emphases on the system goals and implementation process, the actual work is to make module division on a complex system function in accordance with business requirements, establish the hierarchy and calling relationship between modules, determine the interface between modules and the man-machine interface and so on [8], which is the most direct way that the software presents to the user. Users use the system function to complete the daily work and business process, in this process, gradually familiar with the system and evaluate it, according to the business change put forward new requirements, gradually improve the system function. In the design of summary design, we should follow the principles such as abstraction, information hiding, modularization and gradual refinement and so on [9]. Security CT after-sales service system is divided into seven function modules of equipment repair, equipment maintenance, equipment security, spare parts application, spare parts inventory information query, spare parts in-out storage management and basic data management, this paper adopts hierarchy charts to describe the system function structure which is shown in Fig. 2. Main module function descriptions are as follows:

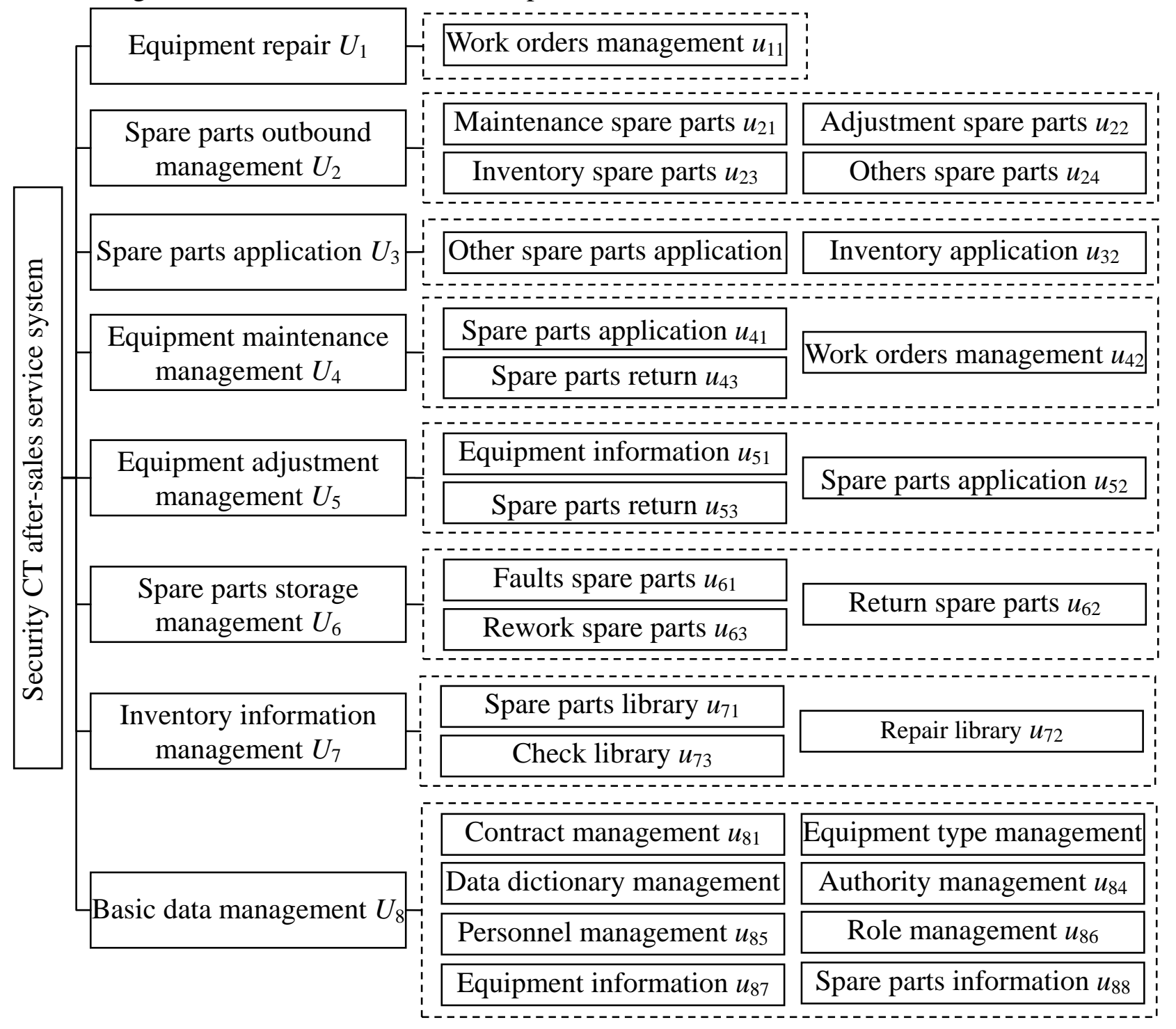

Fig. 2. Security CT after-sales system function structure

First, equipment repair. The main work is to do the repair work order management, record equipment repair basic information, which is the starting point of system business process, all 
operations such as equipment maintenance, spare parts application must be associated with it. Before the repair work order is closed, spare parts can be applied for many times, each work order has one or more maintenance work order.

Second, equipment maintenance. Including maintenance orders management, maintenance spare parts application and return. Maintenance orders management mainly records maintenance related detail information, saves equipment application details, can make CRUD operations on data, but the data can be modified and deleted only in the draft state. Maintenance spare parts application is that maintenance personnel apply required maintenance spare parts according to the experience judgment before going to the scene. Maintenance spare parts return is that maintenance personnel make return application for the spare parts which are not used, after the application form is submitted, which needs to be approved by the competent leadership, once approved, the information needs to be saved in the library to check.

Third, spare parts application. Including other spare parts and stock applications. Other spare parts application is that maintenance personnel loss or damage the rest of spare parts in the maintenance process, unable to return spare parts by the regulation, at this time, filling in the other spare parts application form to explain the reasons. Stock application is to purchase spare parts to prevent that stock spare parts type and quantity are not enough, which affects the daily work.

\section{Data Structure Design}

Database design is the foundation and guarantee of system development. The paper combines the characteristics and business requirements of security CT after-sales service, based on the MySQL database management system, which takes spare parts back inventory management as an example, designs data logic structure, provides technical support for system development.

Return spare parts storage management needs to store return spare parts storage information and identification code material storage information, therefore, the data storage structure is designed for two tables, respectively named as "return spare parts storage application table" and "return spare parts storage application detail table", the primary key words are respectively "return spare parts storage number" and "return spare parts storage detail number" fields, there has a one-to-many relationship between two tables. The spare parts storage application information is saved for a record in the "return spare parts storage application table", however, which contains more than one spare parts detail information, each is not the same, saved for a record in the "return spare parts storage application detail table", so the spare parts detail information are saved as multiple records. As the foreign key of "return spare parts storage application detail table", "Return spare parts storage number" makes associate with "return spare parts storage application table" by the foreign key FK_back_delivery_apply. Based on the above analysis, designed data storage structure and relations based on the MySQL database management system is shown in Fig. 3. 


\begin{tabular}{|c|c|c|}
\hline Back_Delivery_Apply_Table & & Back_Delivery_Apply_Detail_Table \\
\hline $\begin{array}{l}\text { `back_id`varchar(36) not null, } \\
\text { `back_num`varchar(50) default null, } \\
\text { `transactor`varchar(50) default null, } \\
\text { `iven_type`int(11) default null, } \\
\text { `delivery_time`datetime default null, } \\
\text { `note` text, } \\
\text { `attach`text, } \\
\text { ‘create_time`datetime default null, } \\
\text { ‘update_time`datetime default null, } \\
\text { ‘is_del`int(11) default null, } \\
\text { ‘verson`int(11) default null }\end{array}$ & $\begin{array}{l}\text { FK_Back } \\
\text { _Delivery }\end{array}$ & 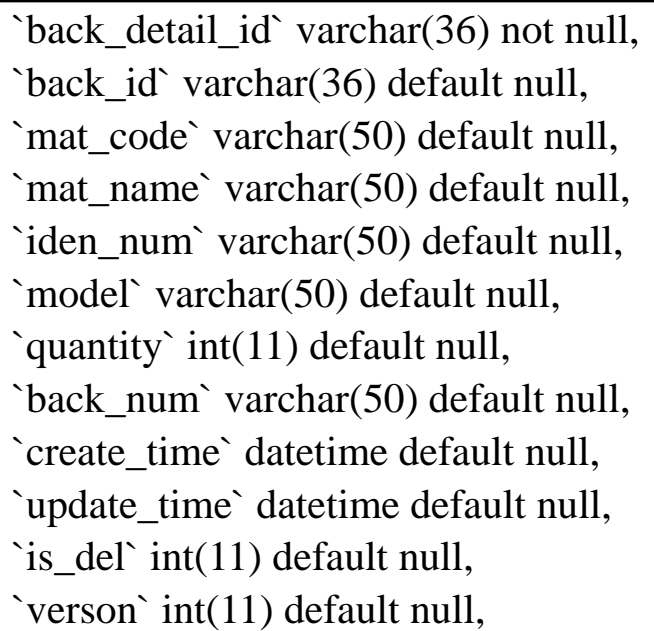 \\
\hline
\end{tabular}

Fig. 3. Data storage structure and relations

In Fig. 3, each table includes four fields of "create_time", "update_time", "delete", and "version". Among them, "create_time" is used to record data creation time, "update_time" is used to record data update time. In order to record the past historical data and facilitate data statistics in the future, using the "delete" field to identify whether this data is logically deleted, rather than physically delete; using the "version" field to identify data version is to consider system upgrade and reform in the future, keep system data.

This paper selects MySQL database management system, which involves three kinds of data types [10]. First, the numerical type, because spare parts are complete, so the number adopts the int type. Second, the character type, which includes a small amount of characters and large texts, a small amount of characters use the varchar type with variable length, large texts select the Text type. Third, the datetime type, MySQL provides five kinds of data types to represent the date time, "storage time" requires to save the specific date time, which chooses the datetime type.

\section{Conclusion}

This article adopts the method and thought of software engineering, based on SSI fusion framework designs security CT after-sales service system, the purpose is to solve the problems encountered in the system development, in order to facilitate the automation, digitization and process management. The security CT after-sales service system built in this paper, the structure is clear, the level is distinct, simple and practical, and can meet the different needs of daily work. Which is conductive to improving the level of after-sales service management, reducing the cost of enterprise operation management, speeding up the speed that staff and leadership get information, truly "information real-time transmission, problems timely processing, risk forecast in advance" [11, 12], drastically enhancing enterprise comprehensive competitiveness.

\section{Acknowledgement}

This work is supported by social science fund project of Liaoning province (L13DTQ01).

\section{References}

[1] S. F. Yin, "Research of Engine Service Management Platform," Manufacturing Automation, vol. 33, no. 9, pp. 21-23, 2011.

[2] H. Hui, L. Ma, "Requirements Analysis Based on UML Online Examination System," Computer Knowledge and Technology, vol. 29, no. 6, pp. 8400-8401, 2010. 
[3] Anne Martens, Heiko Koziolek, "Automatic Model-Based Software Performance Improvement for Component-based Software Designs," Electronic Notes in Theoretical Computer Science, vol. 253, no. 1, pp. 77-93, 2009.

[4] X. Yin, W. Zheng, M. Zhang, "Modularized Operator Interface Framework for Tokamak Based on MVC Design Pattern," Fusion Engineering and Design, vol. 89, no. 5, pp. 628-632, 2014.

[5] L. J. Deng, Y. F. Zhang, J. M. Li, D. X. Dong, "SSI Integration Framework Technology and Application," Journal of Zhengzhou University of Light Industry: Natural Science Edition, vol. 30, no. 1, pp. 46-49, 2015.

[6] J. Meng, "Spring-Java Lightweight Architecture Development Practice," Beijing: The People's Posts and Telecommunications Press, 2006.

[7] Dragos-Paul Pop, Adam Altar. "Designing an MVC Model for Rapid Web Application Development," Procedia Engineering, vol. 69, no. 1, pp. 1172-1179, 2014.

[8] Y. Ma, W. W. Wang, C. Xu, H. D. Li, "Design and Implementation of Web-Based Production Statistic Report," Computer Technology and Development, vol. 22, no. 2, pp. 213-216, 2012.

[9] Y. L. Xiao, "Contract Management System Realization," Computer Programming Skills and Maintenance, vol. 18, no. 14, pp. 21-22, 2011.

[10] L. Huang, D. Y. Liu, "Research on the Public Bicycle Service System by MySQL," Journal of Hubei University for Nationalities: Natural Science Edition, vol. 32, no. 1, pp. 78-80, 2014.

[11] L. Mi, "Demand Analysis in Software Development Cycle," Software Engineer, vol. 3, no. 10, pp. 42-43, 2013.

[12] X. L. Yi, X. Zhang, "Research of Internet Product Requirements Based on Agile Modeling: With Example of Product A," Value Engineering, vol. 31, no. 1, pp. 176-177, 2012. 